\title{
Ventricular Fibrillation Resulting From Ischemia at a Site Remote From Previous Myocardial Infarction
}

\author{
A Conscious Canine Model of Sudden Coronary Death
}

\author{
EUGENE PATTERSON, PhD, KURT HOLLAND, MD, BRIAN T. ELLER, BS, and \\ BENEDICT R. LUCCHESI, MD, PhD
}

In anesthetized dogs, a $\mathbf{3 0}$ gauge silver wire was inserted into the lumen of the left circumflex (LC) coronary artery and myocardial ischemic injury was produced by subsequent occlusion of the left anterior descending (LAD) coronary artery for $90 \mathrm{~min}$ utes followed by reperfusion through a critical stenosis. Four days after acute myocardial infarction, with the dog ambulatory, the intimal surface of the LC coronary artery was injured by applying a $150 \mu \mathrm{A}$ anodal current. Coronary artery thrombosis and subsequent reduction in coronary artery blood flow were accompanied by S-T segment changes at 132 \pm 65 minutes (mean \pm standard deviation [SD]) with ventricular fibrillation (VF) occurring in 29 of 30 dogs $(97 \%)$ at $141 \pm 60$ minutes. Infarct mass in the LAD distribution was $15 \pm 8 \%$ of total left ventricular mass with no histochemical evidence of irreversible ischemic injury in the LC coronary artery distribution. VF was preceded by the development of de- layed electrical activity within the LC coronary artery distribution, and the development of ventricular arrhythmias accompanied by continuous local electrical activity within the subepicardial region of the distribution of the LC coronary artery. In 10 dogs with placement of a critical stenosis around the LAD coronary artery without earlier occlusion and reperfusion, LC intimal injury and subsequent thrombus formation resulted in only 2 deaths $(20 \%)$ from VF. Thus, acute myocardial ischemia at a site distant to a previous myocardial infarction enhances the likelihood of primary VF in the conscious dog. This model of sudden coronary death may simulate the clinical state in man and might serve as an appropriate model for the study of electrophysiologic mechanisms associated with the development of VF and for the evaluation of potential antifibrillatory drugs.
Recent studies by Schuster and Bulkley ${ }^{1}$ demonstrated that acute occlusion of 1 coronary artery could lead to ischemia in the myocardium supplied by a second coronary artery, if the second vessel was critically narrowed and dependent on collateral blood flow from the first coronary artery. These investigators referred to this phenomenon as "ischemia at a distant site," which could contribute to postinfarction angina pectoris and sudden coronary death, an event almost always due to unexpected ventricular tachyarrhythmias. ${ }^{2,3}$ The high rate of recurrence of ventricular fibrillation (VF) and sudden coronary death in patients rescued from a previous event is well documented ${ }^{2,4,5}$ and suggests that the superimposition of an ischemic episode on an already jeopardized myocardium could lead to an arrhythmia terminating in VF.

From the Department of Pharmacology, The University of Michigan Medical School, Ann Arbor, Michigan. This study was supported by Grants HL-05806-21, HL-27817-01, and HL-19782-04 from the National Heart, Lung, and Blood Institute, National Institutes of Health, Bethesda, Maryland. Manuscript received March 9, 1982; revised manuscript received June 2, 1982, accepted June 16, 1982

Address for reprints: Benedict R. Lucchesi, MD, PhD, Department of Pharmacology, M6322 Medical Science Building I, The University of Michigan Medical School, Ann Arbor, Michigan 48109.
There is an urgent need for the development of pharmacologic interventions capable of preventing VF and sudden coronary death in patients identified as being at high risk. One major deficiency in this field of investigation is the lack of a suitable animal model that would permit preclinical studies to identify potentially useful therapeutic agents. Our results support the hypothesis that ischemia at a distant site (circumflex region) in a previously injured myocardium may serve as an important mechanism for the development of ventricular arrhythmias which terminate in sudden and unexpected VF. The experimental model described in this investigation is subject to ventricular tachyarrhythmias in response to programmed electrical stimulation and develops VF in the presence of regional ischemia. Therefore, the model affords the opportunity to initiate meaningful studies aimed at identifying antifibrillatory agents intended to prevent sudden coronary death in man.

\section{Methods}

Surgical Preparation of Dogs With Ischemic Myocardial Injury

Male mongrel dogs (14.6 to $25.6 \mathrm{~kg}$ ) were anesthetized with pentobarbital sodium ( $30 \mathrm{mg} / \mathrm{kg}$ intravenously) and placed 
on positive-pressure respiration using a volume-cycled Harvard pump. Aseptic surgical technique was employed for the insertion of cannulas in the jugular vein and carotid artery, both of which were exteriorized at the back of the neck.

The heart was exposed through a left thoracotomy in the fourth intercostal space. Both the left anterior descending (LAD) and the left circumflex (LC) coronary arteries were freed from the underlying myocardium at a distance of 0.5 to $1.0 \mathrm{~cm}$ from their origin. A 30 gauge Teflon ${ }^{\circledR}$-coated silver wire with $3 \mathrm{~mm}$ of the tip of a 25 gauge hypodermic needle secured on the wire's lead end to aid in penetration of the artery wall, was inserted through the wall of the LC coronary artery so that approximately 2 to $3 \mathrm{~mm}$ of the bare wire-needle tip assembly was within the vessel lumen and in contact with the intimal lining. The wire was secured to the epicardium with 2 sutures. The distal end of the silver wire was exteriorized at the back of the neck and would subsequently serve to deliver an anodal current of $150 \mu \mathrm{A}$ to produce an electrolytic lesion on the intimal surface of the LC coronary artery. A Grass disk electrode was sutured to the subcutaneous tissue and completed the electrical circuit which consisted of a $9 \mathrm{~V}$ battery and a 250,000 $\Omega$ potentiometer placed in a series so as to allow control of the current output at the anode. The procedure was detailed by us in an earlier report. ${ }^{6}$ In 6 of the animals, a calibrated electromagnetic flow probe was placed on the circumflex coronary artery proximal to the point of insertion of the silver wire electrode. The needle-wire assembly was inserted parallel to the inner surface of the LC coronary artery and in the absence of direct flow of current did not result in altered coronary artery blood flow, thrombus formation, or irreversible myocardial ischemic injury in the region of the distribution of the LC coronary vessel, even when left in place for 30 days. ${ }^{6}$

Ischemic myocardial injury was produced in the distribution of the LAD coronary artery as previously described. ${ }^{7}$ This procedure involves the placement of a critical stenosis on the LAD coronary artery by adjusting a ligature tied around the artery and an 18 or 19 gauge needle. The needle is withdrawn and a determination made of the reactive hyperemic response

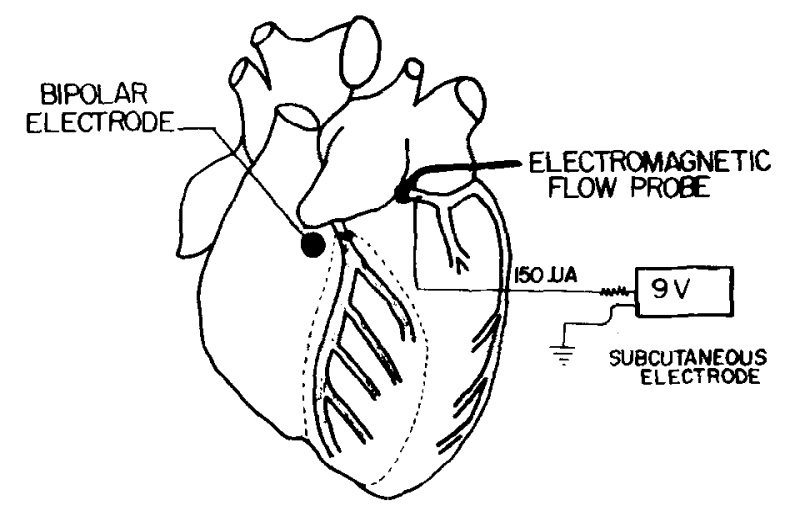

FIGURE 1. Schematic representation of canine heart in which a silver wire electrode is inserted into the LC coronary artery and is attached to the positive pole of a $9 \mathrm{~V}$ nickel-cadmium battery and a variable resistor which permits the current flow to be adjusted to $150 \mu \mathrm{A}$. The negative electrode is attached to the subcutaneous tissue. An electromagnetic flow probe is placed proximal to the intracoronary electrode. The LAD coronary artery has a critical stenosis in its proximai region, is occluded for 90 minutes, and then is reperfused in the presence of the critical stenosis which remains in place until completion of the study. A bipolar electrode in the interventricular septum is used to carry out programmed electrical stimulation after recovery from the operation. Control operated dogs were prepared in a similar manner but did not undergo occlusion of the LAD coronary artery (noninfarct group). to a 10 second occlusion of the LAD vessel. The constricting ligature is adjusted to reduce the reactive hyperemic response by at least $60 \%$, but without reducing resting LAD coronary artery blood flow as recorded by an electromagnetic flow probe placed proximal to the point of vessel constriction. Once partial constriction is established, a Silastic ${ }^{\otimes}$ ligature (Retract-O-Tape is placed distal to the point of constriction and the LAD coronary artery is occluded for 90 minutes followed by reperfusion in the presence of the critical stenosis. The importance of the critical stenosis has been described previously by us $^{7}$ as well as by Sheehan and Epstein ${ }^{8}$ : it reduces the incidence of $\mathrm{VF}$ and the development of hemorrhagic infarct on subsequent reperfusion of the myocardium in the distribution of the LAD coronary artery.

A bipolar plunge electrode consisting of a 25 gauge insulated stainless steel wire, $4 \mathrm{~mm}$ in length and separated by $2 \mathrm{~mm}$, was placed in the ventricular septum adjacent to the right ventricular outflow tract. 'The electrodes were situated $1 \mathrm{~cm}$ from the medial border of the cyanotic area which appeared on occlusion of the LAD coronary artery. Silver disk electrodes were implanted subcutaneously for recording the electrocardiogram (lead II). The surgical incisions were closed, and the animals were given $6 \mathrm{mg} / \mathrm{kg}$ of ampicillin intramuscularly and permitted to recover from anesthesia. Figure 1 is a schematic representation of the surgical preparation of the canine heart in which the area supplied by the LAD coronary artery was subjected to ischemic injury by temporary coronary artery occlusion and reperfusion in the presence of a critical stenosis. In addition, a silver wire electrode was inserted in the LC coronary artery and will be used subsequently to induce an electrolytic lesion on the inner surface of the vessel. The bipolar electrode is to be used for programmed electrical stimulation of the heart and the induction of ventricular arrhythmias.

\section{Experimental Procedures}

Programmed electrical stimulation: The animals were returned to the laboratory after fully recovering from the operation and studied while conscious, unsedated, and resting comfortably in a sling. One, 2 , and 3 ventricular premature stimuli ( $4 \mathrm{~ms}$ duration square-wave pulses, twice diastolic threshold) were applied to the interventricular septum at sequentially decreasing $10 \mathrm{~ms}$ intervals for $S_{2}, S_{3 ;}$, and $S_{4}$ stimuli delivered from a Grass S-88 stimulator and SIU-5 stimulus isolation unit as described previously. ${ }^{9,10}$ The ventricular stimuli from the stimulator were triggered from the $\mathrm{R}$ wave of the lead II electrocardiogram.

The procedure for programmed electrical stimulation can be summarized as follows: (1) Single premature ventricular stimuli $\left(\mathrm{S}_{2}\right)$ at twice diastolic threshold are introduced during normal sinus rhythm at decreasing $10 \mathrm{~ms}$ intervals from 350 ms until ventricular refractoriness occurs. (2) Double premature ventricular stimuli at twice diastolic threshold $\left(S_{2}, S_{3}\right)$ are introduced during normal sinus rhythm at decreasing 10 $\mathrm{ms}$ intervals for $\mathrm{S}_{2}$ stimuli from $350 \mathrm{~ms}$ until ventricular refractoriness occurs with $S_{2}$ to $S_{3}$ intervals of $200,180,170,160$, $150,145,135$, and $130 \mathrm{~ms}$. (3) Triple premature ventricular stimuli at twice diastolic threshold $\left(\mathrm{S}_{2}, \mathrm{~S}_{3,}, \mathrm{~S}_{4}\right)$ are introduced during normal sinus rhythm at decreasing $10 \mathrm{~ms}$ intervals for $S_{2}$ stimuli from $350 \mathrm{~ms}$ until ventricular refractoriness occurs with $S_{2}$ to $S_{33}$ and $S_{33}$ to $S_{4}$ intervals as just stated. (4) Programmed electrical stimulation as just listed is performed during atrial pacing at rates up to 210 beats $/ \mathrm{min}$. (5) Intermittent ventricular pacing at rates up to 360 beats $/ \mathrm{min}$ is performed.

The stimulation protocol was continued until reproducible sustained ventricular tachycardia could be obtained a mini- 
mum of 3 times in each dog. No attempt was made to go beyond this end-point to circumvent the possible initiation of VF.

Initiation of LC coronary artery intimal injury and coronary artery thrombosis: On completion of the programmed electrical stimulation studies, the animals were placed in a quiet laboratory environment and monitored for aberrant electrocardiographic rhythms. The lead II electro cardiogram was transmitted through telemetry and stored on FM magnetic tape for subsequent analysis. Thus, the animals were studied while conscious, ambulatory, and in normal sinus rhythm. The telemetry transmitter and the $9 \mathrm{~V}$ battery source and variable resistor were carried in the pocket of a nylon vest (Alice King Chatham). In 6 animals in which electromagnetic blood flow probes were placed on the LC coronary artery, the study had to be conducted while the conscious, unsedated animals were maintained in a sling, thus limiting their range of movement.

Once adequate baseline recordings were obtained, a continuous direct current anodal current of $150 \mu \mathrm{A}$ was applied to the intimal surface of the LC coronary artery. Previous unpublished studies demonstrated that an anodal current of $150 \mu \mathrm{A}$ would reproducibly lead to oscillatory changes in the LC coronary artery blood flow within 60 to 90 minutes and that an occlusive thrombus would form within 120 to 180 minutes after initiation of current flow. An observer continuously monitored the transmitted data to detect changes in cardiac rhythm, S-T segment shifts, and LC coronary artery blood flow.

Noninfarcted control dogs with LAD coronary artery stenosis and subsequent LC coronary thrombosis: In 10 dogs that served as controls, the LAD coronary artery was constricted so as to minimize or abolish the reactive hyperemic response to a 10 second occlusion of the vessel. Unlike the previous animals in the experimental infarct group, the controls did not undergo temporary occlusion and reperfusion of the LAD coronary artery. Thus, myocardial tissue in the distribution of the vessel was not subjected to ischemic injury. As with the experimental infarct group, however, the control noninfarcted animals were subjected to electrically induced injury to the intimal surface of the LC coronary artery with subsequent formation of an occlusive thrombus and regional

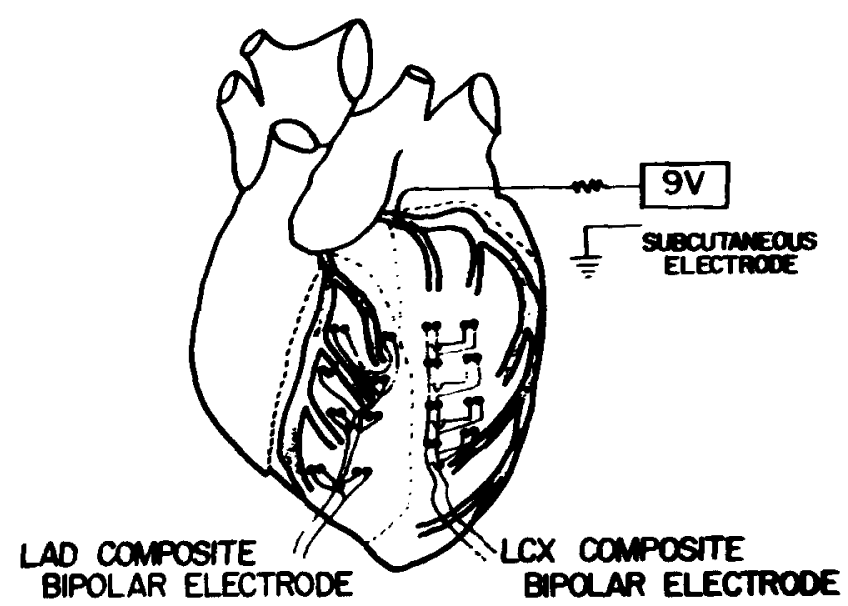

FIGURE 2. Schematic representation of the canine heart similar to that shown in Figure 1, illustrating placement of the bipolar composite electrodes used to record separate subepicardial electrograms from the region of the LAD and the left circumflex (LCX) coronary artery, so that regional electrical events in response to $L C$ coronary artery thrombosis could be studied in the anesthetized open chest dog. myocardial ischemic injury in the distribution of the occluded LC vessel.

Electrophysiologic studies in the anesthetized dog: Male mongrel dogs were prepared in a manner identical to that described for the experimental group $(n=7)$ and for the control noninfarcted group $(n=6)$. 'The animals were returned to the laboratory 4 days after having undergone LAD coronary artcry occlusion and reperfusion in the presence of a critical stenosis. The dogs were anesthetized and placed on positivepressure respiration and the thoracotomy incision was reopened and the heart suspended in a pericardial cradle.

Sixteen pairs of insulated bipolar electrodes $(0.05 \mathrm{~mm}$ in diameter, Teflon ${ }^{\circledast}$-coated silver wire with $2 \mathrm{~mm}$ of exposed tip) were placed subepicardially in the region of distribution of both the I,AD and IC coronary arteries $(3 \times 5 \mathrm{~cm})$. Subepicardial bipolar composite recordings were obtained by summing the 16 bipolar electrode pairs so that separate recordings of composite electrograms could be obtained from each of the regions subserved by the LAD and LC coronary arteries (Fig. 2). The composite electrograms were filtered at 50 and $500 \mathrm{~Hz}$. The electrograms were stored on FM magnetic tape and later recorded on a Visicorder (Honeywell model 1858) along with the lead II electrocardiogram.

Control subepicardial bipolar composite electrode recordings were obtained after which the LC coronary artery was subjected to a continuous $150 \mu \mathrm{A}$ direct current. Measurements of the delay in the subepicardial electrograms were made on a storage oscilloscope (Tektronix model 5111). We quantitated the degree of delay and fragmentation in the subepicardial zone during the induction of thrombus formation and ischemia in the region of distribution of the $\mathrm{LC}$ coronary artery by measuring the maximal delay between the $\mathbf{Q}$ wave of the lead II electrogram and the last distinct and sharply defined activity in the subepicardial composite electrogram during normal sinus complexes. The studies were terminated 8 hours from the start of LC coronary artery simulation or on development of VF.

Quantitation of the extent of irreversible ischemic myocardial injury: At the conclusion of each experiment the heart was removed, examined, and prepared for histochemical staining to identify irreversibly damaged myocardium. The $\mathrm{LC}$ coronary artery was examined for thrombus adherent to the site of intimal injury or in the distal branches of the vessel. The heart was sectioned transversely, apex to base, into $5 \mathrm{~mm}$ thick sections which were incubated at $39^{\circ} \mathrm{C}$ in a $0.5 \%$ solution of triphenyltetrazolium hydrochloride in $0.01 \mathrm{M}$ phosphate buffer at $\mathrm{pH}$ 7.4. The histochemical stain is reduced in the presence of dehydrogenase enzyme activity, thus rendering viable myocardial tissue an intense "brick-red" color and clearly demarcating irreversibly injured tissue which retains a pale coloration. Recent studies ${ }^{11}$ confirm that failure of myocardial tissue to reduce triphenyltetrazolium correlates with the presence of irreversibly injured myocardium and agrees with previous observations from our laboratory. ${ }^{7}$

In the control animals and in the groups used for electrophysiologic studies, thioflavin-S ( $10 \mathrm{ml}$ of a $5 \%$ solution) was injected into the left atrial appendage just before sacrifice by the electrical induction of VF. The heart was removed, sectioned, and stained as described previously. The sections were viewed under ultraviolet light to determine the pattern of distribution of the thioflavin-S which is a flow-dependent fluorescent stain. The appearance of the stain in the region of distribution of the LAD coronary artery was used to confirm the patency of the vessel in which a critical stenosis had been established 4 days earlier. After being viewed under ultraviolet light the ventricular sections were stained in triphenyltetrazolium chloride as described herein.

Statistics: Values for the data are presented as mean \pm 
standard deviation. Fisher's exact tesl was used to analyze the differences in survival between operated control animals and those with experimental infarct. A 2-way analysis of variance was used to analyze for differences in the means between the experimental and the control groups.

\section{Results}

Programmed electrical stimulation in the conscious dog with experimentally induced myocardial infarction ( $L A D$ coronary artery occlusion and reperfusion): Thirty dogs were subjected to temporary occlusion of the I.AD coronary artery and reperfusion in the presence of a critical stenosis, and after full recovery from the operation, the animals were evaluated for the presence of myocardial electrical instability using programmed electrical stimulation. Single, double, and triple premature ventricular stimuli were introduced into the ventricular septum as described previously. Myocardial electrical instability as revealed by electrically inducible ventricular arrhythmias was manifest as either nonsustained ventricular tachycardia in 16 dogs $(53 \%)$, sustained ventricular tachycardia in 7 dogs $(23 \%)$, or VF in 4 dogs $(13 \%)$. Of the 30 animals evaluated on the fourth day after myocardial infarction, $27(90 \%)$ had electrically inducible ventricular arrhythmias in response to programmed cardiac stimulation. The results obtained with programmed electrical stimulation are summarized in Table I and a representative experiment showing the induction of a nonsustained ventricular tachycardia is presented in Figure 3 (top panel). Induced sustained ventricular tachycardia was terminated by rapid ventricular pacing. Once repeatedly initiated nonsustained ventricular tachycardia had been observed in a given animal, using 1 or more modes of atrial or ventricular stimulation, we did not attempt more aggressive stimulation using closer ventricular coupling intervals or faster rates of ventricular
TABLE I Incidence of Myocardial Electrical Instability on Day 4 After Myocardial Infarction

\begin{tabular}{lcccc} 
& \multicolumn{2}{c}{$\begin{array}{c}\text { LAD Coronary Artery } \\
\text { Infarct Group }(\mathrm{n}=30)\end{array}$} & \multicolumn{2}{c}{$\begin{array}{c}\text { Control Group } \\
(\mathrm{n}=10)\end{array}$} \\
\cline { 2 - 5 } & $n$ & $\%$ & $n$ & $\%$ \\
\hline $\begin{array}{l}\text { Not inducible } \\
\text { Nonsustained VT }\end{array}$ & 3 & 10 & 10 & 100 \\
Sustained VT & 16 & 53 & $\ldots$ & $\ldots$ \\
VF & 7 & 23 & $\ldots$ & $\ldots$ \\
\hline
\end{tabular}

* Defibrillated using transthoracic direct current cardioversion (200 to $250 \mathrm{~W}$-s). $\mathrm{VT}=$ ventricular tachycardia.

pacing so as to avoid excess attrition of animals because of electrically induced VF. In the 4 dogs in which VF developed during programmed electrical stimulation, resuscitation was successfully achieved by transthoracic direct current shock.

Programmed electrical stimulation in the conscious dog with an LAD coronary artery critical stenosis, but without myocardial ischemic injury (control, noninfarct group): Programmed electrical stimulation was performed in 10 conscious dogs on the fourth day after placement of a critical stenosis in the LAD coronary artery, but in which myocardial tissue was not subjected to a period of ischemia leading to irreversible injury. Neither nonsustained nor sustained ventricular tachyarrhythmia could be induced in any of the animals through the use of programmed electrical stimulation. Thus, observations in the 10 animals without previously induced ischemic myocardial injury contrast with those obtained in animals that had been subjected to LAD coronary artery occlusion and reperfusion in which myocardial electrical instability could be demonstrated 4 days later in 27 of 30 dogs undergoing provocative electrical stimulation of the heart.
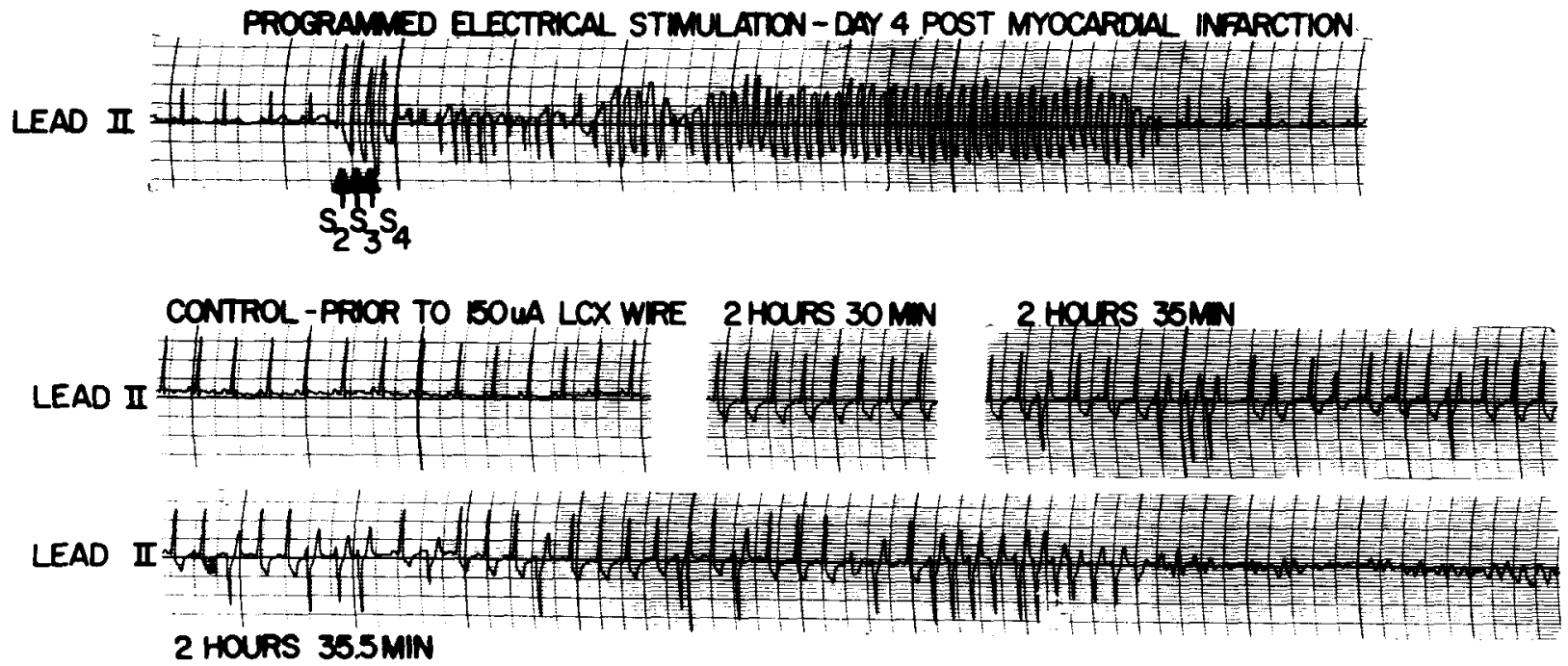

FICURE 3. Ventricular fibrillation in the conscious dog. On day 4 after myocardial infarction, 3 premature ventricular stimuli at twice diastolic threshold are applied to the interventricular septum (coupling intervals of 180, 150, and $150 \mathrm{~ms}$ ) (upper panel). A polymorphic nonsustained ventricular tachycardia is produced. Lead II electrocardiograms before and after stimulation of the intimal surface of the left circumflex (LCX) coronary artery (lower panels) show the development of S-T segment depression followed by disturbances in cardiac rhythm. At 2 hours and 35.5 minutes, the ventricular rhythm degrades to VF. 
TABLE \| Time Course for the Development of Electrocardiographic Changes in the Conscious Dog in Response LC Coronary Artery Thrombosis

\begin{tabular}{lcc}
\hline Days After MI & $\begin{array}{c}\text { LAD Coronary Artery } \\
\text { Infarct Group }(\mathrm{n}=30)\end{array}$ & $\begin{array}{c}\text { Control Noninfarct } \\
\text { Group }(\mathrm{n}=10)\end{array}$ \\
\hline $\begin{array}{l}\text { Minutes to S-T } \\
\text { segment change }\end{array}$ & $132 \pm 65$ & $133 \pm 70$ \\
$\begin{array}{l}\text { Minutes to premature } \\
\text { ventricular } \\
\text { contractions }\end{array}$ & $136 \pm 68$ & $218 \pm 192$ \\
$\begin{array}{l}\text { Minutes to ventricular } \\
\text { tachycardia }\end{array}$ & $138 \pm 58$ & $280 \pm 190$ \\
$\begin{array}{l}\text { Minutes to VT or VF } \\
\text { VF }\end{array}$ & $141 \pm 60$ & 420 \\
\end{tabular}

No significant differences between groups. $\mathrm{MI}=$ myocardial infarction.

Ischemia at a distance (circumflex region) in the dog previously subjected to myocardial ischemic injury and (LAD region) reperfusion: On completion of the protocol for programmed electrical stimulation the animals in both the infarct group $(n=30)$ and the control noninfarct group $(\mathbf{n}=10)$ were subjected to direct anodal current of $150 \mu \mathrm{A}$ applied to the intimal surface of the LC coronary artery through the previously implanted silver wire electrode. The animals were conscious and either unrestrained or limited in mobility by being placed in a sling. As we demonstrated previously, $6,12-14$ electrically induced lesions on the intimal surface of the LC coronary artery result in platelet aggregation and transient oscillation in coronary blood flow associated with changes in the $\mathrm{S}-\mathrm{T}$ segment, ${ }^{14}$ suggesting subendocardial or transmural ischemia.

Continuous monitoring of the lead II electrocardiogram in each of the 30 animals during LC coronary artery stimulation showed that the mean time to initial $\mathrm{S}$-T segment changes $(>0.3 \mathrm{mV})$ was $132 \pm 65$ minutes, with some animals showing $\mathrm{S}$ - T segment depression (n $=25$ ) and others manifesting ischemia by developing $\mathrm{S}$-T segment elevation $(n=5)$. Premature ventricular beats were observed initially at a mean time of $136 \pm 68$ minutes after initiation of current flow to the LC coronary artery with the development of VF at $141 \pm 60$ minutes in 29 of 30 animals $(97 \%)$ with chronic myocardial infarction due to LAD coronary artery occlusion and reperfusion in the presence of a critical stenosis (Table II). All animals were in normal sinus rhythm before the initiation of LC coronary artery stimulation and the development of S-T segment changes in the lead II electrocardiogram. The mean heart rate was 128 \pm 19 beats $/ \mathrm{min}$. With the development of $\mathrm{S}-\mathrm{T}$ segment alterations and presumably ischemia due to changes in LC coronary artery blood flow, the sinus rate increased to $169 \pm 26$ beats $/ \mathrm{min}(\mathrm{p}<0.05)$. Premature ventricular beats or ventricular tachyarrhythmias were not considered in all instances as premonitory signs of VF. In 5 animals, VF was the initial electrocardiographic disturbance associated with the development of S-T segment changes, so that the fatal rhythm developed in the absence of a warning arrhythmia. Figure 3 is taken from a series of electrocardiographic recordings from $1 \mathrm{dog}$ in which nonsustained ventricular tachycardia developed in response to programmed electrical stimulation when tested 4 days after LAD coronary artery occlusion and reperfusion. Subsequent anodal current injury in the $\mathrm{I}, \mathrm{C}$ coronary artery resulted in progressive changes in the electrocardiogram which first showed S-T segment depression and eventually culminated in VF. The time from the first appearance of changes in the S-T segment until the onset of VF showed wide variation among the 30 animals studied. In 6 animals the elapsed time between these events was $<15$ seconds.

In 6 conscious animals, continuous recordings were made of the lead II electrocardiogram, arterial blood pressure, and LC coronary artery blood flow during the induction of low amperage electrical stimulation to the intimal surface of the $\mathrm{LC}$ vessel. The initial LC coronary artery blood flow was $40 \pm 8 \mathrm{ml} / \mathrm{min}$ and the mean arterial blood pressure averaged $101 \pm 9 \mathrm{~mm} \mathrm{Hg}$. LC coronary blood flow was reduced to $4 \pm 6 \mathrm{ml} / \mathrm{min}$ (p $<0.01$ ) and was accompanied by $\mathrm{S}-\mathrm{T}$ segment changes $>0.3 \mathrm{mV}$ with the maximal change occurring at $114 \pm$ 59 minutes after initiating intimal stimulation of the coronary artery. Mean arterial blood pressure was reduced to $87 \pm 9 \mathrm{~mm} \mathrm{Hg}(\mathrm{p}<0.05)$. VF developed in each of the 6 animals within $131 \pm 60$ minutes from the induction of LC coronary artery stimulation. Figure 4 is a representative tracing from 1 of the conscious animals showing the sequence of changes in blood pressure, LC coronary blood flow, and the lead II electrocardiogram during the development of an ischemic episode in the distribution of the LC coronary artery which terminated in VF. In this particular experiment, there was an abrupt cessation of LC coronary artery blood flow, S-T segment depression appeared, and within 15 minutes VF developed.

During these studies the induction of intimal injury to the LC coronary artery was associated with marked alterations in coronary artery blood flow as recorded with an electromagnetic flow probe. Figure 5 is a representative tracing in which arterial blood pressure, the lead II electrocardiogram, and phasic and mean LC coronary artery blood flow are recorded from a conscious dog before and after initiation of current flow to the vessel wall. There is a gradual decrease in amplitude of the phasic coronary blood flow tracing without a change in the mean LC coronary artery blood flow. This is followed by the spontaneous development of a marked reactive hyperemic response (second panel, 1 hour, 9 minutes). Within 10 minutes after the previous record, there is a repetition of the event in which the phasic flow responses are attenuated only to be followed by a reactive hyperemic response. These coronary blood flow changes are cyclic until at 2 hours after the initiation of anodal stimulation of the vessel, coronary flow in the circumflex decreased to 0 and remained at this level until the fibrillation occurred shortly thereafter. Marked disturbances in the electrocardiogram occurred whenever the phasic flow response was attenuated and especially during the development of the reactive hyperemic response. In some instances, VF developed during the active arrhythmic phase associated with the 

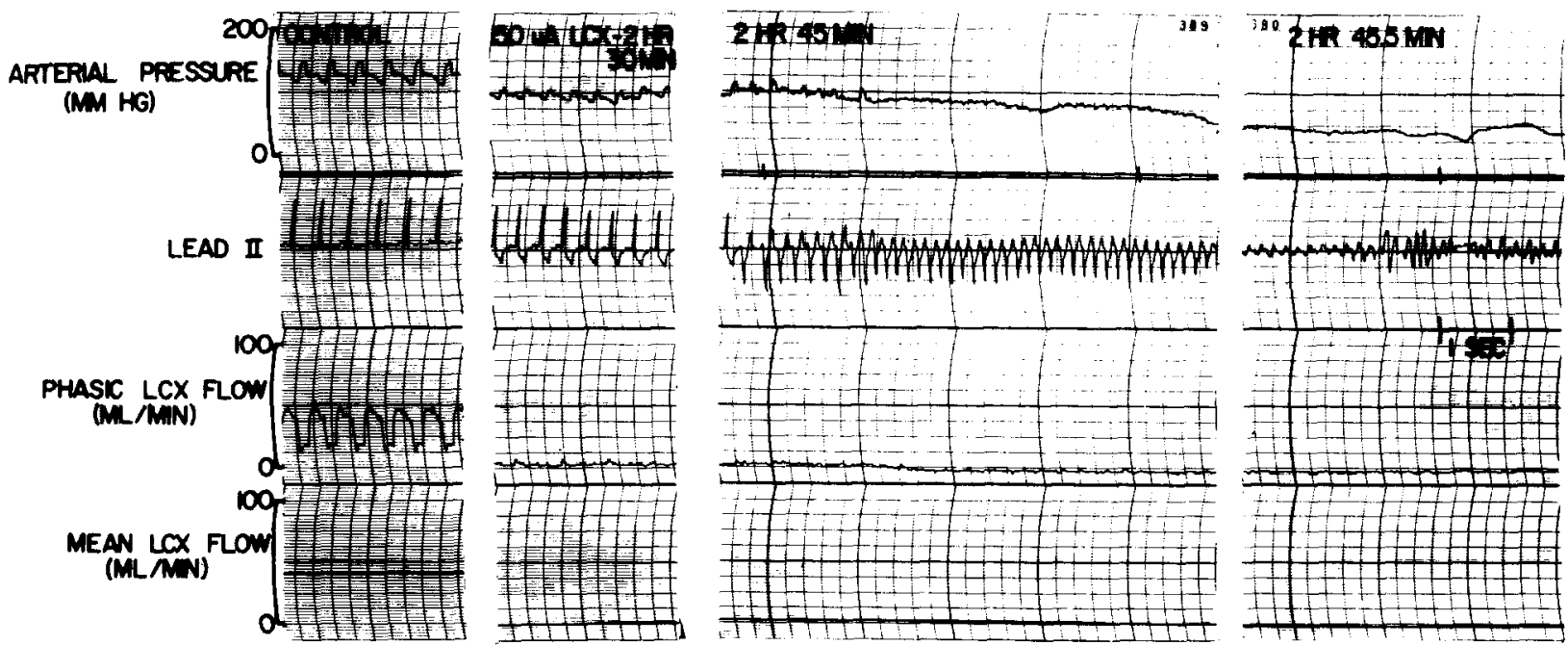

FIGURE 4. Ventricular fibrillation resulting from thrombotic coronary artery occlusion. Arterial pressure tracings, a lead II electrocardiogram, and left circumflex (LCX) coronary blood flow (mean and phasic) are shown before (control) and after stimulation of the intimal surface of the LC coronary artery. At 2.5 hours, S-T segment depression is present in the lead II electrocardiogram and LC coronary artery flow is $0 \mathrm{ml} / \mathrm{min}$. Fifteen $\mathrm{minutes}$ later, sustained VF is observed. An occlusive thrombus, weighing $18 \mathrm{mg}$, was removed from the LC coronary artery at autopsy.

reactive hyperemic response and could account for our inability to identify occlusive thrombi in all animals dying suddenly as a result of VF.

Electrical induction of LC coronary artery thrombosis in the conscious dog with LAD coronary artery stenosis: Ten dogs serving as controls underwent stenosis of the LAD coronary artery, but were noi subjected to occlusion and reperfusion. On the fourth postoperative day, a $150 \mu \mathrm{A}$ anodal current was applied to the intimal surface of the LC coronary artery while the animals were conscious and ambulatory. Changes in the S-T segment, either elevation or depression, occurred at $133 \pm 70$ minutes and this value did not differ significantly from that seen in the previous group with chronic myocardial infarction (Table II). The onset of ventricular ectopic beats and the development of ventricular tachyarrhythmia in response to ischemia in the distribution of the LC coronary artery were delayed in the control group compared with the experimental animals with previous LAD coronary artery occlusion and reperfusion. Although $97 \%$ of the animals with previous myocardial injury developed VF in response to ischemic changes due to alterations in circumflex coronary artery blood flow, only $20 \%$ of the control animals ( $p<0.004$ ) died suddenly as a result of acute ischemia in the distribution of the LC coronary artery. Data for both the experimental infarct group and the control, noninfarct animals are presented in Table II.

Electrophysiologic studies in the anesthetized dog with previous LAD coronary artery occlusion

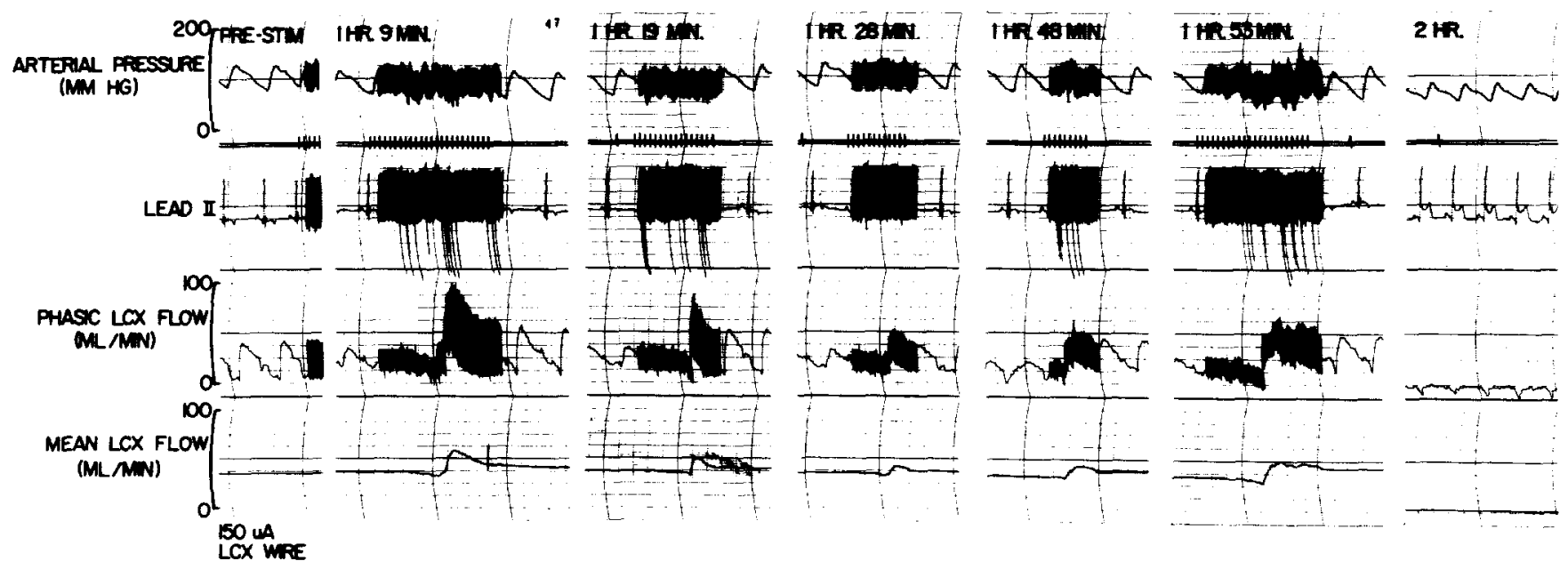

FIGURE 5. Representative recordings of arterial blood pressure, lead II electrocardiogram, and phasic and mean left circumflex (LCX) coronary artery blood flow from a conscious dog that had undergone LAD coronary artery occlusion and reperfusion in the presence of a critical stenosis. The first panel on the left is a control tracing. Subsequent recordings were made after the initiation of LC coronary artery stimulation with a 150 $\mu$ A direct anodal current. See text for description. 
and reperfusion and in control animals without chronic myocardial infarction: The need to obtain electrocardiographic recordings from the subepicardial region of the heart made it necessary to study animals in the anesthetized state and with the heart exposed. Seven dogs with chronic myocardial infarction due to previous LAD occlusion and reperfusion in the presence of a critical stenosis and 6 control dogs with LAD coronary artery stenosis but without chronic myocardial infarction comprised the study groups. Both groups were subjected to intravascular injury in the circumflex coronary artery by application of a $150 \mu \mathrm{A}$ current while subepicardial bipolar composite electrograms were recorded from the respective areas of distribution of the LAD and LC coronary arteries. Recordings of the lead II electrocardiogram and the 2 sets of subepicardial bipolar electrograms were stored on FM magnetic tape and played back on a Honeywell Visicorder. After the control data were obtained, a $150 \mu \mathrm{A}$ anodal current was applied to the LC coronary artery.

Figure 6 is a representative experiment showing the lead II electrocardiogram and separate subepicardial composite electrode recordings from the regions of distribution of the LAD and LC coronary arteries. Composite bipolar electrograms from the LAD and LC coronary artery distributions show discrete deflections without evidence of conduction delay or fragmentation of the normally conducted beats (upper left panel). Despite a region of chronic myocardial injury in the distribution of the LAD artery, there is no evidence of continuous electrical activity during electrical diastole from the subepicardial bipolar composite electrode overlying the area of chronic injury. With the appearance of S-T segment elevation in the lead II electrocardiogram (upper right panel), there is a progressive increase in conduction delay and fragmentation and a decrease in voltage amplitude and appearance of continuous diastolic electrical activity in the composite electrogram recording from the LC coronary artery distribution, whereas the composite bipolar recordings from the region supplied by the LAD coronary artery continue to show a uniform pattern of activation and become disorganized only when there is further disorganization of ventricular electrical activity in the limb lead electrocardiogram. The results in each of the animals with previous LAD coronary artery occlusion and reperfusion and subsequent acute ischemia in the region of the LC coronary artery were similar to those discussed herein (Table III). It was obvious that subepicardial delay of normally conducted beats and persistent diastolic activation developed initially in the region of myocardium supplied by the LC coronary artery and
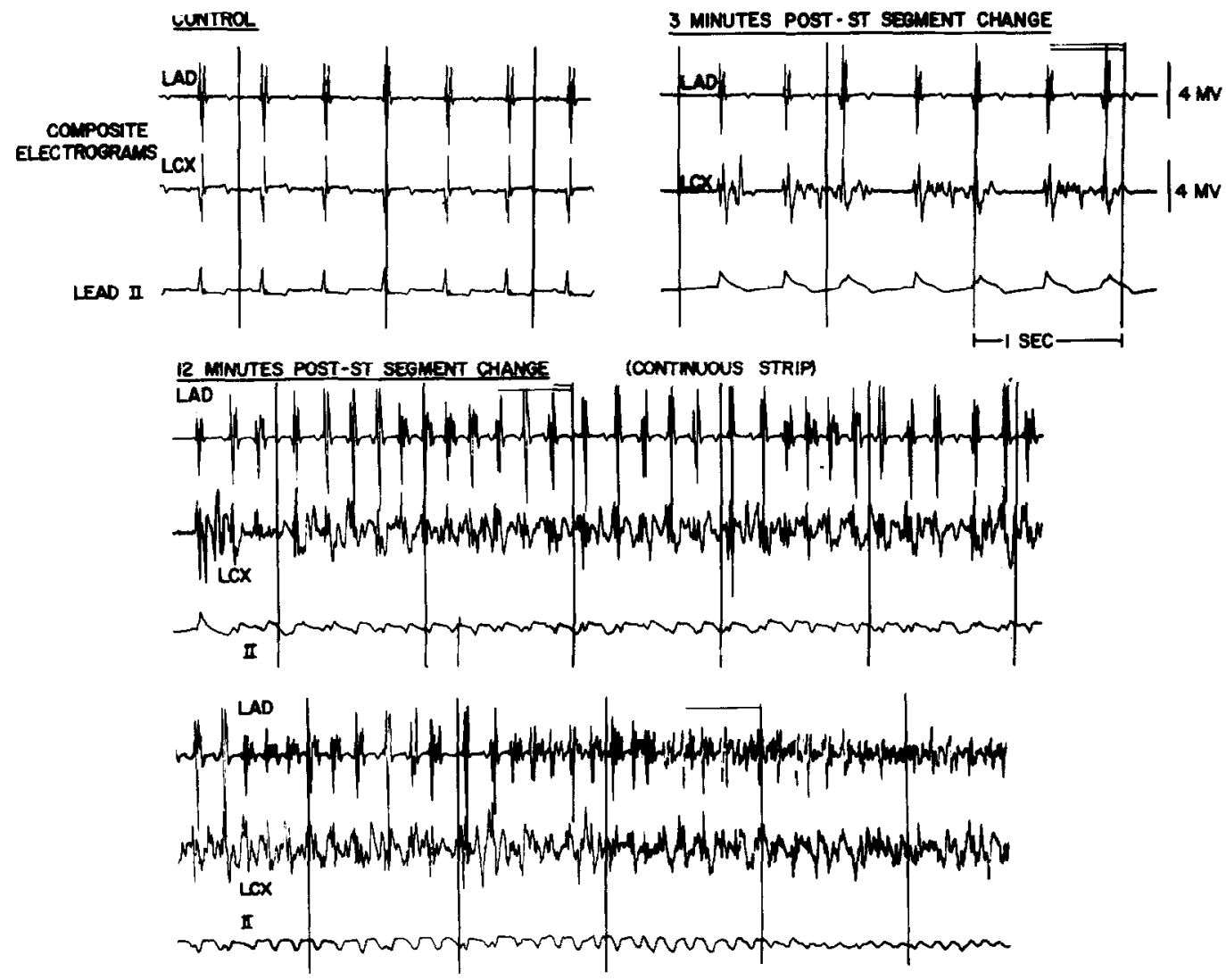

FIGURE 6. Canine model of sudden coronary death. ${ }^{2}$ Control electrograms from the LAD and left circumflex (LCX) distributions are shown in conjunction with a lead II electrocardiogram. Three minutes after S-T segment elevation, delayed and fractionated electrical activity appeared in the circumflex distribution, spanning diastole and producing premature ventricular beats. Twelve minutes after development of S-T segment elevation, a sustained ventricular tachycardia leading to VF was present. Continuous electrical activation within the LC distribution was present from the initiation of tachycardia to VF. 
TABLE III Electrocardiographic Changes in the Anesthetized Canine During Induction of LC Coronary Artery Thrombosis

\begin{tabular}{lcc}
\hline Days After MI & $\begin{array}{c}\text { LAD Coronary Infarct } \\
\text { Group }(n=7)\end{array}$ & $\begin{array}{c}\text { Control Noninfarct } \\
\text { Group }(n=10)\end{array}$ \\
\hline $\begin{array}{l}\text { Minutes to S-T } \\
\text { segment change }\end{array}$ & $98 \pm 58$ & $122 \pm 38$ \\
$\begin{array}{l}\text { Minutes to premature } \\
\text { ventricular beats }\end{array}$ & $108 \pm 55$ & $128 \pm 62$ \\
$\begin{array}{l}\text { Minutes to VT } \\
\text { Minutes to VF }\end{array}$ & $109 \pm 54$ & $196 \pm 80$ \\
\hline
\end{tabular}

No significant differences between day 4 postinfarct group and control group. $\mathrm{Ml}=$ myocardial infarction; $\mathrm{VT}=$ ventricular tachycardia.

was progressive, so that the frequency and severity of the ventricular arrhythmia increased as did the delay in activation in the ischemic heart. Figure 7 shows the changes in epicardial delay as recorded in the experimental infarct animals with previous LAD coronary artery occlusion and reperfusion as compared with dogs with only LAD coronary artery stenosis. The peak epicardial delay in the 2 groups was observed within 10 to 12 minutes after the appearance of S-T segment changes in the lead II electrocardiograms and was significantly greater in the group with previous myocardial ischemic injury as compared with the noninfarcted control animals $(p<0.05)$. Subepicardial composite bipolar electrocardiograms of the region of distribution of the LAD coronary artery in both groups of animals were not altered initially by the subsequent development of ischemia in the myocardial region supplied by the LC coronary artery. In the 7 experimental animals with previous ischemic myocardial injury, VF developed in all after the development of ischemic S-T segment changes in the limb lead electrocardiogram and the appearance of conduction delay and fragmentation in the subepicardial composite bipolar electrograms of the region supplied by the LC coronary artery. However, in only 1 of 6 dogs with LAD coronary artery stenosis and without previous ischemic injury did VF develop in response to ischemia in the myocardial region supplied by the LC coronary artery. Maximal subepicardial delay of normally conducted beats was most pronounced in the group with previous ischemic injury in the region of the LAD coronary artery, and this group displayed a uniform potential to develop VF within 20 minutes after the onset of S-T segment changes (Fig. 7). The development and magnitude of changes in the subepicardial conduction delay appear to be related temporarily to the spontaneous occurrence of ventricular arrhythmias and VF.

Coronary artery thrombosis and irreversible myocardial ischemic injury: VF and sudden death occurred more frequently in those animals subjected to previous LAD coronary artery occlusion and reperfusion. Postmortem examination of the LC coronary artery distal to the point of electrode insertion revealed a thrombus that averaged $11 \pm 7 \mathrm{mg}$. In 5 animals, no evidence of LC coronary artery thrombosis could be found at the site of intimal injury. In 12 animals the
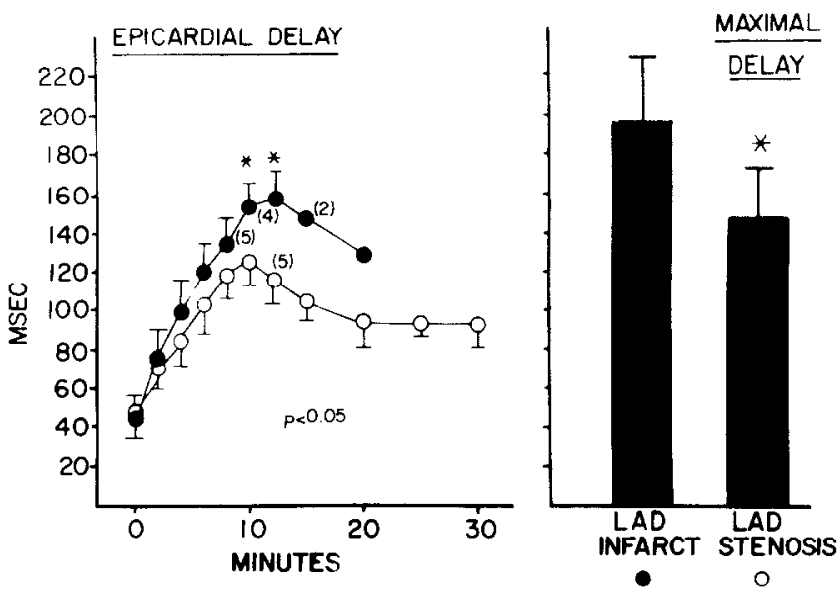

FIGURE 7. Epicardial delays (mean \pm standard deviation) as recorded from the myocardial region supplied by the LC coronary artery in dogs that underwent occlusion and reperfusion of the LAD coronary artery (LAD infarct, $n=7$ ) and in a group of dogs without ischemic injury but with a critical stenosis on the LAD coronary artery (LAD stenosis, $n=$ 6). Myocardial activation time in each group before the development of myocardial ischemia was $<60 \mathrm{~ms}$. The presence of S-T segment changes was accompanied by the development of delayed electrical activation within the LC coronary artery distribution. Delay at 10 and 12 minutes after S-T segment changes was significantly greater in the LAD infarct group. The maximally observed delay for any normal sinus beat in the first 20 minutes after the onset of S-T segment changes was significantly greater in the LAD infarct group.

thrombus averaged less than $5 \mathrm{mg}$. Complete occlusion of the lumen of the LC artery by the thrombus was not observed in 18 of 30 animals dying suddenly. Because of instantaneous death in this group and because ischemia in the distribution of the LC existed only briefly before the onset of VF, postmortem staining of the ventricular sections with triphenyltetrazolium chloride did not reveal any evidence of irreversible ischemic injury in the LC distribution. However, because animals in the infarct group had been subjected to previous occlusion and reperfusion of the LAD coronary artery, triphenyltetrazolium staining showed an anterior wall infarct that averaged $15 \pm 8 \%$ of total left ventricular mass.

In the noninfarcted control dogs subjected to LAD coronary artery stenosis, subsequent induction of intimal injury of the LC coronary artery produced a mean thrombus of $48 \pm 40 \mathrm{mg}$ (range 4 to $135 \mathrm{mg}$ ) and in 9 of 10 animals resulted in total occlusion of the vessel. The thrombus was significantly larger $(p<0.05)$ in the control group, probably because 8 of 10 animals survived 24 hours, allowing time for continued development and organization of an obstructive lesion. In only 2 of the 10 animals in this group did VF develop (Table II). Postmortem examination in the remaining 8 dogs 24 hours later showed an infarct mass in the distribution of the circumflex coronary artery which averaged $18 \pm 12 \%$ of the total left ventricle. In the control group there was no evidence of myocardial tissue injury in the region of distribution of the stenosed LAD coronary artery. Furthermore, the administration of thioflavin-S just before sacrifice of the animals resulted in perfusion of the anterior wall segment supplied by the stenosed 
vessel. Thus, despite total thrombotic occlusion of the LC coronary artery, in the presence of a critical stenosis in the LAD coronary vessel, VF or sudden death occurred in only $20 \%$ of the animals as compared with $97 \%$ of the animals that had chronic myocardial infarction due to previous ischemic injury resulting from occlusion and reperfusion of the LAD coronary vessel. In the latter group, fatal VF occurred despite the fact that the LC coronary artery did not always undergo total occlusion. Thus, a brief ischemic episode due to alterations in coronary artery blood flow in a region remote from a previous myocardial infarction is capable of leading to a rhythm disturbance that has the potential to initiate VF and sudden coronary death.

\section{Discussion}

Coronary artery disease is responsible for over $90 \%$ of sudden deaths occurring each year in the general population. In a study by Lie and Titus, ${ }^{15} 62$ to $74 \%$ of the hearts of victims of sudden and unexpected death showed evidence of acute or old myocardial infarction. Coronary artery stenosis was present in the heart in over $90 \%$ of these victims, and specialized staining methods provided evidence of acute myocardial ischemia in up to $81 \%$ of the hearts examined. McQuay et $a^{16}$ suggested that postinfarction angina might occur and contribute to death in as many as $20 \%$ of patients with fatal infarcts. The recent and elegant studies by Schuster and Bulkley ${ }^{1}$ add further support to the concept that ischemia at a site remote from a previous myocardial infarction could contribute to sudden coronary death.

The animal model described in this report demonstrates that an acute ischemic event, when superimposed on a ventricular myocardium with a history of previous ischemic injury, can lead to an electrophysiologic disturbance culminating in VF. However, myocardial ischemia in the distribution of the LC coronary artery in the absence of a previous myocardial infarction carried a low risk of lethal arrhythmia, suggesting that disorganization of cardiac rhythm is more likely to occur in a ventricle that serves as a proper substrate for initiating and maintaining a rhythm capable of degenerating into VF. Through the use of programmed electrical stimulation, we as well as others have demonstrated that occlusion and reperfusion of the LAD coronary artery renders the canine heart susceptible in the long term to electrical induction of nonsustained or sustained ventricular tachycardia, or both.9,10,17 Histologic examination of the myocardium subjected to occlusion and reperfusion showed viable myocardium within the necrotic region, suggesting that salvaged myocardial fibers may provide reentrant pathways causing sustained tachyarrhythmias. ${ }^{17-20}$ Previous experimental and clinical studies have provided sufficient evidence that ventricular tachyarrhythmias in response to programmed electrical stimulation are due to reentry and less likely to be related to triggered automaticity or to other electrophysiologic mechanisms. ${ }^{21-26}$ Data in the present study agree with previously published observations on the reentrant nature of induced arrhythmias.
The existence of conduction defects in the epicardium of the infarcted canine heart after experimentally induced myocardial infarction has been described ${ }^{24,27}$ and shown to be associated with reentrant ventricular arrhythmias which can be induced using the same techniques of programmed electrical stimulation practiced in the clinical laboratory. Thus, the animal model in which myocardial ischemic injury has resulted from occlusion and reperfusion of the LAD coronary artery renders the heart highly susceptible to sustained ventricular tachyarrhythmias which can be terminated by single or multiple electrical stimuli.

From our observations we assume that electrically induced arrhythmia arises from myocardium within or adjacent to the infarct and depends on the heterogeneous nature of the damaged tissue due to reperfusion after a period of ischemia. ${ }^{17-20}$ 'This assumption is supported by our ability to regularly record the presence of continuous electrical activity originating from the ischemic zone ( $\mathrm{LC}$ region) and bridging the diastolic interval between the initiating and reentrant beats as well as between consecutive reentrant beats. However, this disturbance alone does not explain why the chronically damaged heart is, for a variable period of time, susceptible to the development of VF. Neither we nor others ${ }^{17-20}$ have noted any great tendency for VF to develop in the chronically damaged heart during programmed electrical stimulation in which the provocative stimuli were limited to variables that produced sustained or nonsustained ventricular tachycardia, or both. The addition of a second, albeit remote, ischemic episode due to initiation of an occlusive thrombotic event in the LC coronary artery causes an additional region of the heart to support a reentry pattern which ultimately engages the chronically injured region, thus leading to VF. It appears as if a critical mass of myocardium capable of providing a region of sufficient length to support a reentrant pathway is essential for the development and maintenance of spontaneous VF. Furthermore, regional myocardial ischemia as well as decreased collateral blood flow to the area of chronic myocardial infarction would result from a disturbance in LC coronary artery blood flow. Myocardial ischemia would decrease the threshold for the development of VF, thereby enhancing the possibility of fatal arrhythmia. In addition, tachyarrhythmia resulting from ischemia in the region of the LC coronary artery would further enhance the tendency for VF to develop. ${ }^{28}$

Our evidence illustrates that electrically induced injury to the LC causes regional disturbances in coronary blood flow, resulting in ectopic ventricular activity associated with continuous electrical activity as recorded with subepicardial bipolar composite electrodes in the acutely ischemic $L C$ region. The bridging of the diastolic interval was associated with ventricular tachycardia due most likely to a reentrant mechanism as discussed herein and supported by the elegant observations of El-Sherif et al. ${ }^{24,29}$ Subsequent to this event and just before the onset of VF, continuous diastolic electrical activity developed within the region of the LAD coronary artery. One might assume that overlapping of the 2 regions of reentry would result in 
a relatively large zone of diastolic electrical activation capable of sustaining the reentrant mechanism leading to VF. This concept that a sufficient reentrant zone is required for the development of $\mathrm{VF}$ is supported by our observation that thrombotic occlusion of the LC coronary artery in the presence of LAD coronary artery stenosis, but without a previous myocardial infarction, seldom resulted in VF. This might be explained on the basis of a lack of a critical mass of myocardium with altered electrophysiologic properties capable of initiating and sustaining VF. Thus, the addition of a remote site of ischemia in the presence of chronic myocardial infarction may be a major factor leading to the development of VF.

We are well aware that not all authorities would agree with the interpretation of our results and the analysis of the subepicardial electrograms. We suggest that although continuous electrical activity is expected within an area of reentry, continuous activity is not, in itself, proof of reentry. ${ }^{30,31}$ In contrast to many previous studies, we employed a composite electrode made up of an array of subepicardial recording sites. Furthermore, the electrical event being recorded is that which precedes the development of $\mathrm{VF}$ and is in response to an acute ischemic episode which in the majority of instances was transmural myocardial ischemia due to interruption in LC coronary artery blood flow. Using a subepicardial bipolar composite electrode recording arrangement similar to that described herein, Kaplinsky et $\mathrm{al}^{32}$ analyzed the exact time course of ventricular arrhythmias after coronary artery ligation and correlated these events with the local disturbance of activation in the ischemic zone. Their data show that VF of immediate (first 10 minutes) ventricular arrhythmias is associated with severe subepicardial delay of the normally conducted beats, whereas VF of the delayed (12 to 30 minutes) arrhythmias was not associated with subepicardial delay. The development of VF in the model described herein is closely related to that of the immediate variety discussed by Kaplinsky et al. 32

It is difficult to extrapolate from the animal experiment to the clinical situation. However, the animal model of "sudden coronary death" presented in this study may provide a useful tool to explore in greater detail those electrophysiologic events associated with the development of $\mathrm{VF}$ in a totally physiologic setting not requiring anesthesia and not being dependent on the application of external electrical stimui to initiate and sustain the development of VF. Furthermore, we view this animal model as a potentially useful adjunct in the final preclinical evaluation of pharmacologic interventions designed to prevent VF. 33

\section{References}

1. Schuster EH, Bulkley BH. Ischemia at a distance after acute myocardial infarction: a cause of early post-infarction angina. Circulation 1980;62 509-515.

2. Liberthson RR, Nagel EL, Hirschman JC, Nussenfeld SR. Pre-hospital ventricular defibrillation: prognosis and follow-up course. N Engl J Med 1974;291:317-321.

3. Klein RC, Vera Z, Mason DT, DeMaria AN, Awan NA, Amsterdam EA. Ambulatory Holter monitor documentation of ventricular tachyarrhythmias as mechanism of sudden death in patients with coronary artery disease (abstr). Clin Res 1979;27:7A

4. Cobb LA, Baum RS, Alverez H III, Schaffer WA. Resuscitation from outof-hospital ventricular fibrillation: 4 years follow-up. Circulation 1975;51, 52:Suppl III:III-223

5. Schaffer WA, Cobb LA. Recurrent ventricular fibrillation and modes of death in survivors of out-of-hospital ventricular fibrillation. N Engl J Med 1975; 293:259-262

6. Romson JL, Haack DW, Lucchesi BR. Electrical induction of coronary artery thrombosis in the ambulatory canine: a model for in vivo evaluation of anti-thrombotic agents. Thromb Res 1980:17:841-853.

7. Lucchesi BR, Burmeister WE, Lomas TE, Abrams GD. Ischemic changes in the canine heart as affected by the dimethyl quaternary analog of proin the canine heart as affected by the dimethyl quaternary analog of propran

8. Sheehan FH, Epstein SE. Determinants of arrhythmic death due to coronary spasm: pre-existing coronary stenosis reduces risk of VF following release of coronary occlusion. Circulation 1982;65:259-264.

9. Patterson E, Lucchesi BR. Chronic ventricular tachyarrhythrnias in the conscious dog: prevention by UM-272 (dimethylpropranolol). J Cardiovasc Pharmacol 1981;3:769-780.

10. Patterson E, Gibson JK, Lucchesi BR. Chronic canine ventricular tachyarrhythmias: prevention by bretylium tosylate administration. Circulation arrhythmias: prevention

11. Fishbein MC, Meerbaum S, Rit J, Lando U, Kanmatsuse K, Mercier JC, Corday E, Ganz W. Early phase acute myocardial infarct size quantification: validation of the triphenyl tetrazolium chloride tissue enzyme staining technique. Am Heart J 1981;101:593-600.

12. Romson JL, Bush LR, Haack DW, Lucchesi BR. The beneficial effects of oral ibuprofen on coronary artery thrombosis and myocardial ischemia in the conscious dog. J Pharmacol Exp Ther 1980;215:271-278.

13. Romson JL, Haack DW, Abrams GD, Lucchesi BR. The prevention of occlusive coronary artery thrombosis by prostacyclin $\left(\mathrm{PGl}_{2}\right)$ infusion in the dog. Circulation 1981;64:906-914.

14. Romson J, Lucchesi BR. Experimental myocardial ischemia provoked by platelet aggregation and coronary artery spasm. In: Rafflenbeul W, Lichtlen PR, Balcon R, eds. Unstable Angina Pectoris. New York: Georg Thieme Verlag, 1981:13-17.

15. Lie JT, Titus JL. Pathology of the myocardium and the conduction system in sudden coronary death (abstr). Circulation 1975;51:Suppl III:|ll-41.

16. McQuay NW, Edwards SE, Burchell MB. Types of death in acute myocardial infarction. Arch Intern Med 1955:96:1-10

17. Karagueuzian HS, Fenuglio JJ Jr, Weiss MB, Wit AL. Protracted ventricular tachycardia induced by premature stimulation of the canine heart after coronary artery occlusion and reperfusion. Circ Res 1979;44:833-846.

18. Gibson JK, Lucchesi BR. Effect of pranolium on ventricular tachycardia induced in canine myocardium after myocardial infarction (abstr). Circulation 1978;57,58:Suppl III:III-45

19. Gibson JK, Lucchesi BR. Electrophysiologic actions of UM-272 (Pranolium) on reentrant ventricular arrhythmias in post-infarction canine myocardium. J Pharmacol Exp Ther 1980;214:247-253.

20. Michelson EL, Spear JF, Moore EN. Electrophysiologic and anatomic correlates of sustained ventricular tachyarrhythmias in a model of chronic myocardial infarction. Am J Cardiol 1980;45:583-590.

21. Wellens HJJ, Lle KI, Durrer D. Further observations on ventricular tachycardia as studied by electrical stimulation of the heart. Chronic recurrent ventricular tachycardia and ventricular tachycardia dur ing acule myocardial infarction. Circulation 1974;49:647-653.

22. Wit AL, Bigger JT Jr. Possible electrophysiological mechanisms for tetha arrhythmias accompanying myocardial ischemia and infarction (abstr). Circulation 1975;51-53:Suppl ill:lli-96.

23. Bassett AL, Gelband H, Nilsson K, Morales AR, Myerburg RJ. Electrophysiology following healed experimental myocardial infarction. In: Kulbertus physiology following healed experimental myocardial infarction. In: Kulbertus University Park Press, 1976:242-255.

24. El-Sherif N, Scherlag BJ, Lazzara R, Hope RR. Reentrant arrhythmias in the late myocardial infarction period. I. Conduction characteristics in the infarct zone. Circulation 1977;55:686-702.

25. Josephson ME, Horowilz LN, Farshidi A, Spielman SR, Michelson EL, Greenspan AM. Sustained ventricular tachycardia: evidence for protected localized reentry. Am J Cardiol 1978;42:416-424

26. Hope RR, Scherlag BJ, Lazzara R. Excitation of ischemic myocardium altered properties of conduction, refractoriness, and excitability. Am Heart altered properties of

27. Hope RR, Scherlag BJ, Lazzara R, El-Sherif N. Ventricular arrhythmias in healing myocardial infarction: role of rhythm versus rate in reentrant activation. J Thorac Cardiovasc Surg 1978;75:458-466

28. James RGG, Arnold JMO, Allen JD, Pantridge JF, Shanks RG. The effects of heart rate, myocardial ischemia and vagal stimulation on the threshold for ventricular fibrillation. Circulation 1977:55:311-317.

29. El-Sherif N, Hope RR, Scherlag BJ, Lazzara R. Reentrant ventricular arrhythmias in the late myocardial infarction period. 2 . Patterns of initiation and termination of reentry. Circulation 1977;55:702-719.

30. Janse MJJ, van Capelle FJL, Morsink H. Flow of "injury" current patterns of excitation during early ventricular arrhythmias in acute regional myocardial ischemia in isolated porcine and canine hearts: evidence for two cardial ischemia in isolated porcine and canine hearts: evidence for
different arrhythmogenic mechanisms. Circ Res 1980;47:151-165.

31. Wit AL, Allessie MA, Bonke FIM, Lammers W, Smeets J, Fenoglio JJ. Electrophysiologic mapping to determine the mechanism of experimental ventricular tachycardia initiated by premature impulses. Am $J$ Cardiol 1982;49:166-185

32. Kaplinsky E, Ogawa S, Balke CW, Dreifus LS. Two periods of early ventricular arrhythmias in the canine acute myocardial infarction model. Circulation 1979;60:397-403.

33. Holland K, Patterson E, Lucchesi BR. Prevention of ventricular fibrillation by bretylium in a conscious canine model of sudden coronary death. Am Heart $J 1982$, in press. 